Is the $\mathrm{CMB}$ cold spot a gate to extra dimensions?

This article has been downloaded from IOPscience. Please scroll down to see the full text article.

JCAP10(2008)039

(http://iopscience.iop.org/1475-7516/2008/10/039)

View the table of contents for this issue, or go to the journal homepage for more

Download details:

IP Address: 147.96.14.15

The article was downloaded on 04/02/2013 at 16:39

Please note that terms and conditions apply. 


\title{
Is the CMB cold spot a gate to extra dimensions?
}

\author{
J A R Cembranos ${ }^{1}$, A de la Cruz-Dombriz ${ }^{2}$, \\ A Dobado ${ }^{2}$ and A L Maroto ${ }^{2}$ \\ ${ }^{1}$ William I. Fine Theoretical Physics Institute, University of Minnesota, \\ Minneapolis, MN 55455, USA \\ 2 Departamento de Física Teórica I, Universidad Complutense de Madrid, \\ E-28040 Madrid, Spain \\ E-mail: cembranos@physics.umn.edu,dombriz@fis.ucm.es, dobado@fis.ucm.es \\ and maroto@fis.ucm.es
}

Received 29 July 2008

Accepted 5 October 2008

Published 24 October 2008

Online at stacks.iop.org/JCAP $/ 2008 / \mathrm{i}=10 / \mathrm{a}=039$

doi:10.1088/1475-7516/2008/10/039

\begin{abstract}
One of the most striking features found in the cosmic microwave background data is the presence of an anomalous cold spot (CS) in the temperature maps made by the Wilkinson Microwave Anisotropy Probe (WMAP). This CS has been interpreted as the result of the presence of a collapsing texture, perhaps coming from some early universe grand unified theory (GUT) phase transition. In this work we propose an alternative explanation based on a completely different kind of texture which appears in a natural way in a broad class of brane-world models. This type of texture, known as a braneskyrmion, can be understood as a hole in the brane which makes it possible to pass through them along the extra-dimensional space. The typical scales needed for the proposed brane-skyrmions to correctly describe the observed CS can be as low as the electroweak scale.
\end{abstract}

Keywords: CMBR theory, extra dimensions, cosmology with extra dimensions, cosmological applications of theories with extra dimensions 
One of the most important pieces of information about the history and nature of our universe comes from the cosmic microwave background (CMB). Measurements of the CMB temperature anisotropies obtained by WMAP $[1,2]$ have been thoroughly studied in recent years. Such anisotropies have been found to be Gaussian, as expected in many standard cosmological scenarios corresponding to density fluctuations of one part in a hundred thousand in the early universe. However, by means of a wavelet analysis, an anomalous CS, apparently inconsistent with homogeneous Gaussian fluctuations, was found in $[3,4]$ centred at the position $b=-57^{\circ}, l=209^{\circ}$ in galactic coordinates, with a characteristic scale of about $10^{\circ}$. The existence of this CS has been confirmed more recently in $[5,6]$.

What is the origin of this CS? A very interesting possibility has been proposed recently in [7]. According to it some theories of high energy physics predict the formation of various types of topological defects, including cosmic textures [8], which would generate hot and cold spots in the CMB [9]. These textures would be the remnants of a symmetry breaking phase transition that took place in the early universe. In order to produce textures, the cosmic phase transition must be related to a global symmetry breaking pattern from one group $G$ to a subgroup $H$ so that the coset space $K=G / H$ has a non-trivial third homotopy group. A typical example is $K=S U(N)$, which has associated $\pi_{3}(K)=\mathbb{Z}$ for $N \geq 2$. Notice that, as usual, in order to get a texture formed in the transition, the symmetry breaking must correspond to a global symmetry since if it were local it could be gauged away.

Textures can be understood as localized wrapped field configurations which collapse and unwind on progressively larger scales. Textures can produce a concentration of energy which give rise to a time-dependent gravitational potential. CMB photons traversing the texture region will suffer a red- or a blueshift producing a cold or hot spot in the CMB maps. In [7] the authors consider a $S U(2)$ Nonlinear Sigma Model (NLSM) to build up a model of texture that could explain the observed CS at $b=-57^{\circ}, l=209^{\circ}$. They simulate the unwinding texture by using a spherically symmetric scaling solution and they find a fractional temperature distortion given by

$$
\frac{\Delta T}{T}(\theta)= \pm \epsilon \frac{1}{\sqrt{1+4\left(\theta / \theta_{\mathrm{c}}\right)^{2}}}
$$

where $\theta$ is the angle from the centre, $\epsilon$ is a measure of the amplitude and $\theta_{c}$ is the scale parameter that depends on the time at which the texture unwinds. The best fit of the CS is found for $\epsilon=7.7 \times 10^{-5}$ and $\theta_{\mathrm{c}}=5.1^{\circ}$. In the above solution, this value for $\theta_{\mathrm{c}}$ implies that the observed texture unwound at $z \sim 6$. On the other hand, the parameter $\epsilon$ is given by $\epsilon \equiv 8 \pi^{2} G \Phi_{0}$, where $\Phi_{0}$ is the fundamental symmetry breaking scale which is then set to be $\Phi_{0} \simeq 8.7 \times 10^{15} \mathrm{GeV}$. This scale is nicely close to the GUT scale, thus making the results given in [7] extremely interesting.

Nevertheless it is important to stress that textures require having a global symmetry breaking but what one finds typically in GUTs is a local symmetry breaking producing the Higgs mechanism and then destroying the topological meaning of the texture or any other possible defect appearing in the cosmic transition. In this work we explore a different possibility to obtain textures that could explain the CS. This possibility is based on the so-called brane-world models which have the advantage of providing global symmetries by assuming proper boundary conditions and symmetries for the fields propagating in the bulk space. In these models, our universe is considered to be a three-dimensional brane 
with tension $\tau \equiv f^{4}$ living in higher $(D=4+N)$-dimensional space (the bulk space) with the $N$ extra dimensions compactified to some manifold $B$ with small volume $V_{N}[10]$. Then it is possible to find the relation $M_{\mathrm{P}}^{2}=V_{N} M_{D}^{2+N}$, where $M_{\mathrm{P}}$ is the Planck scale and $M_{D}$ is the fundamental gravity scale in $D$ dimensions which, in the phenomenologically interesting cases, could be of the order of $\mathrm{TeV}$ (the electroweak scale). In this kind of scenario, the Standard Model (SM) particles are not allowed to leave the brane, but gravitons propagate through the whole bulk space. For simplicity we will assume that the whole $D$-dimensional space can be factorized as $M_{D}=M_{4} \times B$ with the brane lying on the $M_{4}$ space-time manifold (for simplicity we will assume $B$ to be homogeneous). Then we can choose the obvious coordinates $\left\{x^{\mu}, y^{m}\right\}$ with $\mu=0,1,2,3 ; m=1,2, \ldots, N$ and the ansatz for the bulk metric $G_{M N}=\operatorname{diag}\left(\tilde{g}_{\mu \nu}(x), \tilde{g}_{m n}^{\prime}(y)\right)$. In the absence of the brane, this metric possesses an isometry group that is assumed to be of the form $G\left(M_{D}\right)=G\left(M_{4}\right) \times G(B)$. The presence of the brane spontaneously breaks the symmetry to some subgroup $G\left(M_{4}\right) \times H$, with $H \subset G(B)$ being some subgroup of $G(B)$. The position of the brane can be parametrized as $\left\{x^{\mu}, Y^{m}(x)\right\}$ and its induced metric in the ground state is just $g_{\mu \nu} \equiv \tilde{g}_{\mu \nu} \equiv G_{\mu \nu}$. However, when brane excitations (branons) are present, the induced metric is given by $g_{\mu \nu}=\tilde{g}_{\mu \nu}-\partial_{\mu} Y^{m} \partial_{\nu} Y^{n} \tilde{g}_{m n}^{\prime}$. On the other hand, it is easy to show that $B$ is diffeomorphic to the coset $K=G(B) / H$ which is the space of the Goldstone bosons (GB) associated with the spontaneous symmetry breaking of the isometries - transverse translation-produced by the presence of the brane. Thus the transverse translations of the brane - branons - can be considered as GB on the coset $K$ and the branon fields can be defined as coordinates $\pi^{\alpha}$ on $K$ as: $\pi^{\alpha}=\left(v / R_{B}\right) \delta_{m}^{\alpha} y^{m}$, where $R_{B}$ is the typical size of the compactified space and $v \equiv f^{2} R_{B}$ is the typical size of the coset $K$. Then, the induced metric on the brane can be written in terms of the branon fields $\pi$ as

$$
g_{\mu \nu}=\tilde{g}_{\mu \nu}-\frac{1}{f^{4}} \partial_{\mu} \pi^{\alpha} \partial_{\nu} \pi^{\beta} h_{\alpha \beta}
$$

where $h_{\alpha \beta}$ is the $K$ metric which can be obtained easily from the $B$ metric [11]. In more complicated cases, the metric $g_{\mu \nu}$ could also be a function of the extra dimension coordinates $y$. Then it is possible to show that branons may become massive. In fact, in [12] these massive branons were shown to behave as weakly interacting massive particles (WIMPs) and thus being natural candidates for dark matter in this kind of scenario.

However, in this work we would like to study another important property of the branon fields, namely the possibility of wrapping around the extra dimension space $B$ giving rise to non-trivial topological configurations as was studied in detail in [13]. In particular, for $\pi_{3}(B)=\pi_{3}(K)=\mathbb{Z}$ we may have texture-like configurations called braneskyrmions. As we will see these brane-skyrmions have some common features with the textures considered in [7] and therefore could provide an explanation of the CS observed in the CMB in these kinds of extra-dimensions brane-world models.

Brane-skyrmions can be nicely understood in geometrical terms as some kind of holes in the brane that make it possible to pass through them along the $B$ space. This is because in the core of the topological defect the symmetry is re-established. In particular, in the case considered in this work the broken symmetry is basically the translational symmetry along the extra dimensions. 
At low energies, brane dynamics can be described by the Nambu-Goto action:

$$
S=-f^{4} \int \mathrm{d}^{4} x \sqrt{-g}
$$

with $g_{\mu \nu}$ being the induced metric defined in (2). In order to simplify the calculations we will consider the simplest case $B \simeq K \simeq S U(2) \simeq S^{3}$ and introduce spherical coordinates on both spaces, $M_{4}$ and $K$. In $M_{4}$ we denote the coordinates $\{t, r, \theta, \varphi\}$ with $\phi \in[0,2 \pi)$, $\theta \in[0, \pi]$ and $r \in[0, \infty)$. On the coset manifold $K$, the spherical coordinates are denoted $\left\{\chi_{K}, \theta_{K}, \phi_{K}\right\}$ with $\phi_{K} \in[0,2 \pi), \theta_{K} \in[0, \pi]$ and $\chi_{K} \in[0, \pi]$. Notice that such coordinates cover the whole spherical manifolds and relate to the physical branon fields (local normal geodesic coordinates on $K$ ) by

$$
\begin{aligned}
& \pi_{1}=v \sin \chi_{K} \sin \theta_{K} \cos \phi_{K}, \\
& \pi_{2}=v \sin \chi_{K} \sin \theta_{K} \sin \phi_{K}, \\
& \pi_{3}=v \sin \chi_{K} \cos \theta_{K} .
\end{aligned}
$$

The coset metric in spherical coordinates is written as

$$
h_{\alpha \beta}=\left(\begin{array}{ccc}
v^{2} & & \\
& v^{2} \sin ^{2}\left(\chi_{K}\right) & \\
& & v^{2} \sin ^{2}\left(\chi_{K}\right) \sin ^{2}\left(\theta_{K}\right)
\end{array}\right) .
$$

In terms of branon fields, it can be shown that the Nambu-Goto action can be expressed as a derivative expansion, whose lowest-order term with two field derivatives is nothing other than $S U(2)$ NLSM. In spherical coordinates, the brane-skyrmion with winding number $n_{W}$ is given by the non-trivial mapping $\pi^{\alpha}: S^{3} \longrightarrow S^{3}$ defined from

$$
\phi_{K}=\phi, \quad \theta_{K}=\theta, \quad \chi_{K}=F(t, r),
$$

with boundary conditions satisfying $F(t, \infty)-F(t, 0)=n_{W} \pi$. This map is usually referred to as the hedgehog ansatz.

From the Nambu-Goto action, we obtain the equation of motion for our skyrmion profile $F(t, r)$ :

$$
\begin{aligned}
\sin (2 F)-2 r F^{\prime} & +\left(r^{2}+\frac{v^{2}}{f^{4}} \sin ^{2} F\right) \\
& \times \frac{\ddot{F}-F^{\prime \prime}+\left(v^{2} / f^{4}\right)\left(\ddot{F} F^{2}+F^{\prime \prime} \dot{F}^{2}-2 F^{\prime} \dot{F} \dot{F}^{\prime}\right)}{1-\left(v^{2} / f^{4}\right)\left(\dot{F}^{2}-F^{\prime 2}\right)}=0
\end{aligned}
$$

where the dot and prime denote $t$ and $r$ derivatives, respectively.

In this work we are interested in the potential cosmological effects due to the presence of a brane-skyrmion within our Hubble radius. For that purpose we will compute the gravitational field perturbations at large distances compared to the size of the extra dimensions, i.e. we are interested in the $r^{2} \gg R_{B}^{2}=v^{2} / f^{4}$ region. Notice that, in this region, gravity behaves essentially as in four-dimensional space-time and standard General Relativity can be used in the calculations. Notice also that, in order to simplify the calculations, we will ignore the effects due to the universe expansion. This is justified provided $r \ll H_{0}^{-1}$. In such a case the unperturbed (ignoring the defect presence) background metric can be taken as Minkowski, i.e. $\tilde{g}_{\mu \nu}=\eta_{\mu \nu}$, and the above equation of 
motion reduces to

$$
r^{2}\left(\ddot{F}_{0}-F_{0}^{\prime \prime}\right)+\sin \left(2 F_{0}\right)-2 r F_{0}^{\prime}=0
$$

which is equivalent to expression (3) in [9]. Notice that this is an expected result since, as shown in $[8,14]$, at large distances, i.e. except in the microscopic unwinding regions, the dynamical evolution of the fields are completely independent of the symmetry breaking mechanism, it simply depends on the geometry of the coset manifold $K$. On small scales $[8,14]$ it is possible that higher-derivative terms could affect the dynamics and even stabilize the textures. This is also the case of brane-skyrmions [13], although generically they could unwind by means of quantum-mechanical effects.

Equation (8) admits an exact solution with $n_{W}=1$ :

$$
F_{0}(t, r)=2 \arctan (-r / t)
$$

with $t<0$ since, in order to get a cold spot, photons should pass the texture position before collapse.

Our approximated equation (8) is consistent with (7) for the above solution since the second term in the numerator vanishes for $F \equiv F_{0}$ and

$$
\begin{aligned}
& \dot{F}_{0}^{2}-F_{0}^{\prime 2}=4\left(r^{2}-t^{2}\right) /\left(r^{2}+t^{2}\right)^{2}, \\
& \sin ^{2} F_{0}=4 r^{2} t^{2} /\left(r^{2}+t^{2}\right)^{2},
\end{aligned}
$$

so that, in the considered regime, the neglected terms are irrelevant for all $r$ and $t$ values.

Once $F(t, r)$ has been determined, we may calculate the energy-momentum tensor components also in this region from the Nambu-Goto action as: $T^{\mu \nu}=-2|\tilde{g}|^{-1 / 2} \delta S / \delta \tilde{g}_{\mu \nu}$. In spherical coordinates they become

$$
\begin{array}{ll}
T_{00}=\frac{2 v^{2}\left(r^{2}+3 t^{2}\right)}{\left(t^{2}+r^{2}\right)^{2}} ; & T_{r r}=\frac{2 v^{2}\left(r^{2}-t^{2}\right)}{\left(t^{2}+r^{2}\right)^{2}} ; \\
T_{0 r}=-\frac{4 v^{2} r t}{\left(t^{2}+r^{2}\right)^{2}} ; & T_{\theta \theta}=\frac{2 v^{2} r^{2}\left(r^{2}-t^{2}\right)}{\left(t^{2}+r^{2}\right)^{2}} ; \\
T_{\phi \phi}=\sin ^{2} \theta T_{\theta \theta} . &
\end{array}
$$

Note that $\nabla_{\mu} T_{\nu}^{\mu}$ identically vanishes. We will now determine the background metric $\tilde{g}_{\mu \nu}$ in the $r \gg R_{B}$ region and in the presence of the brane-skyrmion as a small perturbation on the Minkowski metric, i.e. $\tilde{g}_{\mu \nu}=\eta_{\mu \nu}+h_{\mu \nu}$.

Thus, for the scalar perturbation of the Minkowski space-time in the longitudinal gauge we have

$$
\mathrm{d} s^{2}=(1+2 \Phi) \mathrm{d} \eta^{2}-(1-2 \Psi) \delta_{i j} \mathrm{~d} x^{i} \mathrm{~d} x^{j}
$$

where potentials $\Phi \equiv \Phi(t, \vec{x})$ and $\Psi \equiv \Psi(t, \vec{x})$ and perturbed Einstein's tensor components in Cartesian coordinates are the following (see [15]):

$$
\begin{aligned}
\delta \mathcal{G}_{0}^{0} & =2 \nabla^{2} \Psi \\
\delta \mathcal{G}_{i}^{j} & =\left[-2 \ddot{\Psi}-\nabla^{2}(\Phi-\Psi)\right] \delta_{i}^{j}+\partial_{i} \partial^{j}[\Phi-\Psi], \\
\delta \mathcal{G}_{i}^{0} & =2 \partial_{i} \dot{\Psi},
\end{aligned}
$$


with $i, j=1,2,3$ and $\nabla^{2} \equiv \sum_{i=1}^{3} \partial_{i} \partial^{i}$. Using the Einstein equation $\delta \mathcal{G}_{\nu}^{\mu}=8 \pi \mathrm{G} T_{\nu}^{\mu}$ we determine that perturbed quantities $\Phi$ and $\Psi$ are

$$
\Psi \equiv \Phi=4 \pi \mathrm{G} v^{2} \log \frac{r^{2}+t^{2}}{t^{2}} .
$$

The physical metric on which photons propagate is not the $\tilde{g}_{\mu \nu}$ we have just calculated, but the induced metric (2). However, using the solution in (9), we find that the contribution from branon fields is $\mathcal{O}\left(R_{B}^{2} / r^{2}\right)$, i.e. negligible, so that $g_{\mu \nu} \simeq \tilde{g}_{\mu \nu}$.

Photons propagating on the perturbed metric will suffer a red-(blue)shift due to the Sachs-Wolfe effect. The full expression for the temperature fluctuation is given by

$$
\left(\frac{\Delta T}{T}\right)_{\mathrm{SW}}=-[\Phi]_{\tau_{i}}^{\tau_{f}}+\int_{\tau_{i}}^{\tau_{f}}(\dot{\Psi}+\dot{\Phi}) \mathrm{d} \tau=-[\Phi]_{\tau_{i}}^{\tau_{f}}+\int_{\tau_{i}}^{\tau_{f}} 2 \dot{\Phi} \mathrm{d} \tau
$$

where we have considered local and integrated SW effects and neglect the Doppler contribution. $\tau_{i}$ is the decoupling time and $\tau_{f}$ is the present time. Substituting expression (14) in the previous one and using that $r^{2}=z^{2}+R^{2}$, and $z=t-t_{0}$, we get

$$
\left(\frac{\Delta T}{T}\right)_{\mathrm{SW}}=8 \pi \mathrm{G} v^{2}\left[\frac{t_{0}}{\sqrt{2 R^{2}+t_{0}^{2}}} \arctan \frac{t_{0}+2 z}{\sqrt{2 R^{2}+t_{0}^{2}}}-\log \left|t_{0}+z\right|\right]
$$

where $R$ is the impact parameter and $t_{0}$ is the time at which the photon passes the texture position at $z=0$. In the limit where $z_{i} \rightarrow-\infty$ and $z_{f} \rightarrow \infty$ the result is

$$
\left(\frac{\Delta T}{T}\right)_{\mathrm{SW}}=\epsilon \frac{t_{0}}{\sqrt{2 R^{2}+t_{0}^{2}}}
$$

with $\epsilon \equiv 8 \pi^{2} \mathrm{G} v^{2}$, in complete agreement with [9].

Let us estimate the scales required for this kind of texture to explain the observed CS. For the minimal model supporting brane-skyrmions with three extra dimensions $N=3$ we will have $M_{\mathrm{P}}^{2} \simeq R_{B}^{3} M_{D}^{5}$, with $M_{\mathrm{P}}$ the Planck mass, $M_{D}$ the fundamental scale of gravitation in $D=7$ dimensions and $R_{B}^{3}$ the characteristic volume for the compactified extra dimensions. Therefore

$$
R_{B} \simeq\left(\frac{M_{\mathrm{P}}^{2}}{M_{D}^{5}}\right)^{1 / 3}
$$

and consequently

$$
v^{2} \equiv f^{4} R_{B}^{2} \simeq f^{4}\left(\frac{M_{\mathrm{P}}^{2}}{M_{D}^{5}}\right)^{2 / 3} .
$$

In order to get $\epsilon \simeq 7.7 \times 10^{-5}$, we need $v \simeq 1.2 \times 10^{16} \mathrm{GeV}$, which in fact can be achieved with $M_{D} \sim f \sim \mathrm{TeV}$. Notice that, for this parameter range, the radius of the extra dimension is around $R_{B} \sim 10^{-8} \mathrm{~m}$, i.e. our approximations are totally justified. In addition, we have also checked that the possible effects coming from a non-vanishing branon mass are suppressed by $\mathcal{O}\left(M^{2} / v^{2}\right)$. Therefore, for mass values also around $M \sim \mathrm{TeV}$ (which are typical of branon as dark matter, see [12]) such effects are negligible. 
In other words, brane-skyrmions provide an accurate description for the CS without the need for introducing very high energy (GUT) scales since the correct temperature fluctuation amplitude can be obtained with natural values around the electroweak scale.

Let us emphasize that there is no difference between the scenario where branons behave like WIMPs and the one containing brane-skyrmions, except that the latter is a subset of the former, since not all the brane-worlds support textures. In other words, the same model can provide at the same time WIMPs in the form of branons and braneskyrmions able to explain the cold spot. This is quite remarkable, since it allows us to explain both phenomena with the same scale, which may be as low as the electroweak scale.

In order to estimate the abundance of brane-skyrmions in this model, it is important to take into account that their low energy (large distance) dynamics is described by the same NLSM as that in [7] and therefore the expected evolution of textures in an expanding universe in the model presented in this work will be completely equivalent to that in the mentioned reference. This is nothing other than the well-known fact that, except in the microscopic unwinding region, the field evolution only depends on the geometry of the coset space $K$, but not on the details of the symmetry breaking mechanism. As shown in [14] in a simple model with a potential term, the final abundance of defects and other properties of the pattern of density perturbations are expected to be not very sensitive to the short distance physics, once the texture unwinds, making this kind of theory highly predictive. For that reason, we expect that, provided the same kind of initial conditions are imposed in both models, the predicted abundance of hot and cold spots agree with that obtained from simulations in [7]. Such simulations show that the number of textures unwindings per comoving volume and conformal time $\eta$ can be estimated as $(\mathrm{d} n / \mathrm{d} \eta)=\nu \eta^{-4}$ with $\nu \simeq 2$. This allows us to estimate the number of hot and cold spots in a given angular radius interval. As commented above, this is quite a robust result at late times with little effects from short distance dynamics. In the case of brane-skyrmions, the short distance effects will be embodied in the higher-derivative terms appearing in the expansion of the Nambu-Goto action or even in possible induced curvature terms generated by quantum effects [13]. Provided that such terms do not stabilize the braneskyrmions, we expect that such an abundance could be directly applied in our case.

Future experiments will be able to confirm the studied model. On the one hand, the fact that the fundamental scales of the theory are of the order of $\mathrm{TeV}$ opens up the possibility to test this explanation with collider experiments through the production of real or virtual branons and KK-gravitons. The expected signatures of the model [16] from the production of KK-gravitons come fundamentally from the single-photon channel studied by LEP, which restricts $M_{D}>1.2 \mathrm{TeV}$ at $95 \%$ of C.L. On the other hand, the LHC will be able to test the model up to $M_{D}=3.7 \mathrm{TeV}$, analysing single-photon and monojet production.

Finally, there also exists the possibility to find signatures of the model at low energies associated with the branon (brane fluctuations) phenomenology. This case is more interesting from the cosmological point of view, since branons can constitute the non-baryonic dark matter abundance as typical WIMPs $[12,17]$. Present constraints coming from the single-photon analysis realized by L3 (LEP) imply $f>122 \mathrm{GeV}$ (at $95 \%$ C.L.) $[18,19]$ and the LHC will be able to check this model up to $f=1080 \mathrm{GeV}$ through monojet production [17]. The idea to test the physics associated with the cold spot 
with the next generation of colliders at the $\mathrm{TeV}$ scale is a very intriguing and distinctive property of this texture.

\section{Acknowledgments}

This work has been partially supported by the DGICYT (Spain) under projects FPA200402602, FPA2005-02327 and CAM/UCM 910309, by UCM-Santander PR34/07-15875 and by DOE grant DOE/DE-FG02-94ER40823.

\section{References}

[1] Spergel D N et al, 2007 Astrophys. J. Suppl. 170377

[2] Hinshaw et al, 2007 Astrophys. J. Suppl. 170288

[3] Vielva P, Martínez-González E, Barreiro R B, Sanz J L and Cayón L, 2004 Astrophys. J. Suppl. 60922

[4] Cruz M, Martínez-González E, Vielva P and Cayón L, 2005 Mon. Not. R. Astron. Soc. 36529

[5] Cruz M, Cayón L, Martínez-González E, Vielva P and Jin J, 2007 Astrophys. J. 65511 [SPIRES]

[6] Naselsky P D et al, 2007 arXiv:0712.1118 [astro-ph]

[7] Cruz M, Turok N, Vielva P, Martinez-Gonzalez E and Hobson M, 2007 Science 3181612

[8] Turok N, 1989 Phys. Rev. Lett. 632625 [SPIRES] Turok N, 1991 Phys. Scr. T 36135

[9] Turok N and Spergel D, 1990 Phys. Rev. Lett. 642736 [SPIRES]

[10] Arkani-Hamed N, Dimopoulos S and Dvali G, 1998 Phys. Lett. B 429263 [SPIRES] Antoniadis I et al, 1998 Phys. Lett. B 436257 [SPIRES]

[11] Dobado A and Maroto A L, 2001 Nucl. Phys. B 592203 [SPIRES]

[12] Cembranos J A R, Dobado A and Maroto A L, 2003 Phys. Rev. Lett. 90241301 [SPIRES] Cembranos J A R, Dobado A and Maroto A L, 2003 Phys. Rev. D 68103505 [SPIRES]

[13] Cembranos J A R, Dobado A and Maroto A L, 2002 Phys. Rev. D 65026005 [SPIRES]

[14] Spergel D N et al, 1991 Phys. Rev. D 431038 [SPIRES]

[15] Giovannini M, 2005 Int. J. Mod. Phys. D 14363 [SPIRES]

[16] Yao W M et al (Particle Data Group), 2006 J. Phys. G: Nucl. Part. Phys. 331 [SPIRES] Mirabelli E A, Perelstein M and Peskin M E, 1999 Phys. Rev. Lett. 822236 [SPIRES]

[17] Cembranos J A R, Dobado A and Maroto A L, 2004 Phys. Rev. D 70096001 [SPIRES]

[18] Alcaraz J et al, 2003 Phys. Rev. D 67075010 [SPIRES]

[19] Achard P et al (L3 Collaboration), 2004 Phys. Lett. B 597145 [SPIRES] 